\title{
Wind Turbine Interference in a Wind Farm Layout Optimization Mixed Integer Linear Programming Model
}

\author{
Rosalind Archer', Gary Nates ${ }^{2}$, Stuart Donovan ${ }^{3}$ and Hamish Waterer 4 \\ 1,2,3 Department of Engineering Science, University of Auckland, Private Bag 92019, Auckland, New \\ Zealand \\ ${ }^{4}$ School of Mathematical and Physical Sciences, University of Newcastle, University Drive, \\ Callaghan NSW 2308, Australia \\ 'communicating author, e-mail <r.archer@auckland.ac.nz> \\ 2e-mail <gnat007@aucklanduni.ac.nz> \\ 3e-mail <stu.donovan@gmail.com> \\ ${ }^{4}$ e-mail <hamish.waterer@newcastle.edu.au>
}

\begin{abstract}
This work develops a wind intensity interference coefficient which captures the interference caused by an upwind turbine on a downwind turbine in the same wind flow. This coefficient includes the use of a Weibull distribution to handle variability in the wind velocity, and also accounts for the geometric relationship between the turbines and the boundaries of the wind sector. This interference coefficient then forms part of a mixed integer linear program (MILP) which is used to optimise the locations of wind turbines within a wind farm site. The MILP approach is an exact method that can guarantee that the turbine locations determined by the model are optimal with respect to an a priori chosen set of possible turbine locations. Tests on an example data set (based on a demonstration case in the WindFarmer software) show, for the particular wind resource used, that interference results in a loss of power of approximately $4.2 \%$ when compared to the power production which would be predicted without accounting for interference.
\end{abstract}

\section{NOMENCLATURE}

A

$c_{t}$

$D$

$d$

F

$h$

I

$L$

$P$

area swept by turbine rotors, $\mathrm{m}^{2}$

thrust coefficient, dimensionless

diameter of the rotor blades, $\mathrm{m}$

wind direction

Weibull distribution

turbine height, $m$

wind intensity interference coefficient, MWh

turbine location causing interference

power, MWh

origin / location of the upwind turbine

the radial distance to downwind turbine with respect to a turbine at the origin, $m$

set of possible wind directions

axial thrust, $\mathrm{N}$ 


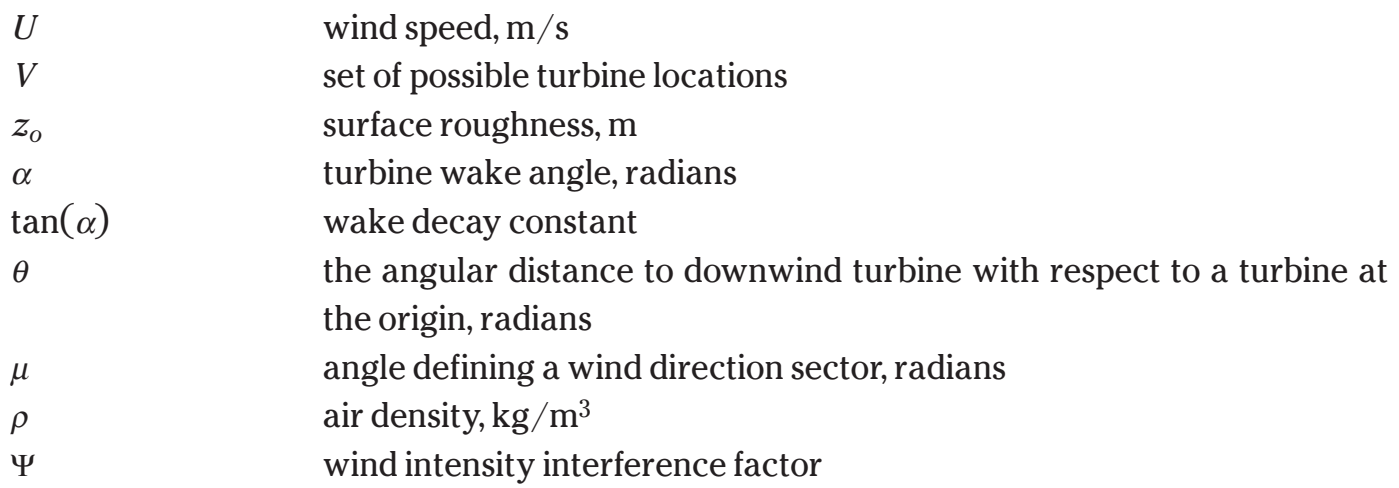

\section{INTRODUCTION}

When wind flows through a wind turbine, there is a transfer of kinetic energy from the wind to the rotors of the turbine, which causes a reduction in the wind speed. As the wind flows further downstream from the turbine, the wind speed gradually returns to its original level.

Assume two turbines are placed in front of one another and the wind flows in a straight line through each turbine. The turbine downwind will experience a reduction in the incoming wind speed compared with the wind speed the same turbine would experience if there were no turbine located upwind. The area downwind of a turbine which experiences this reduction in wind speed and produces interference is known as the turbine wake. This wind speed deficit is known as interference. The modified PARK model (GH, 2006 and Jensen, 1983) describes the turbine wake and finds the reduced wind speed. This model assumes a rectangular wind speed profile and the wake expands linearly behind the rotor. This is shown in Figure 1.

Equation 1 defines the modified PARK wind turbine wake model and relates the wind velocity at location $i\left(U_{i}\right)$ to the wind velocity at location $j\left(U_{j}\right)$.

$$
U_{j}=U_{i}\left[1-\left(1-\sqrt{1-c_{t}}\right)\left(\frac{D}{D+2 r \tan (\alpha)}\right)^{2}\right]
$$

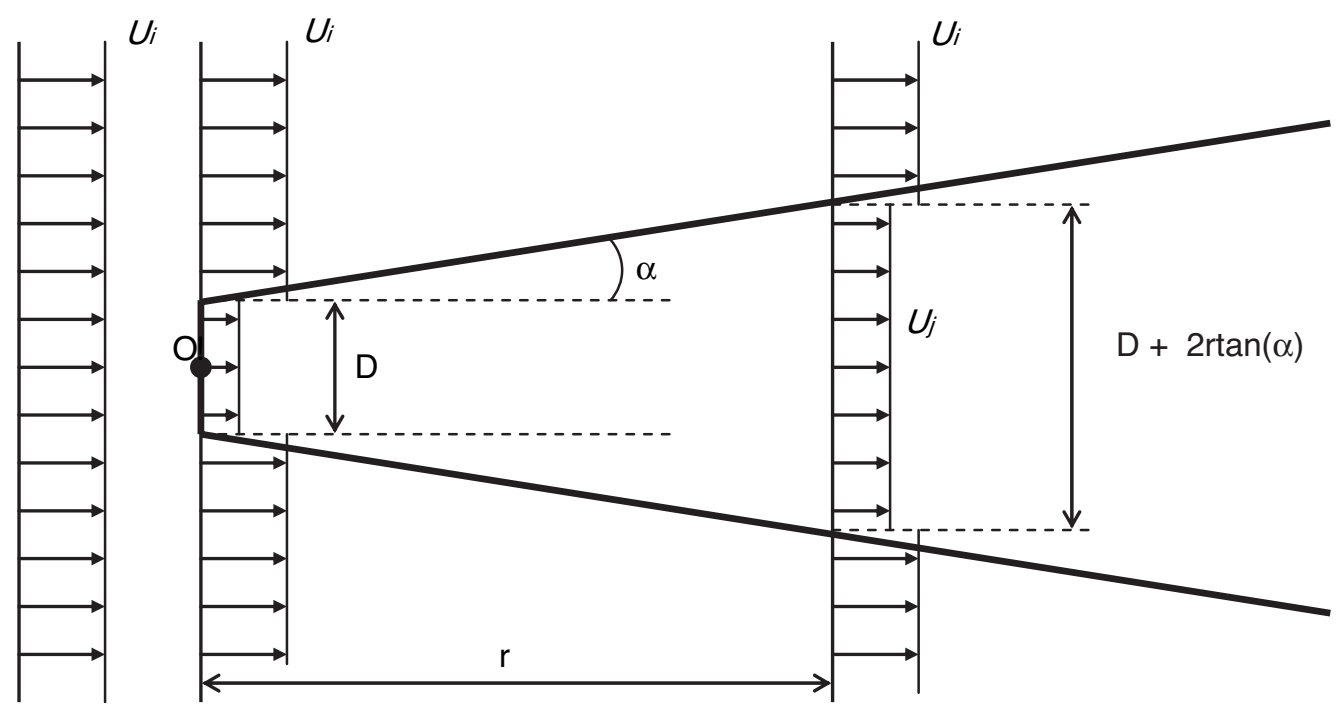

Figure 1: Schematic of the modified PARK model (aerial view) (after Hansen, 2007) 


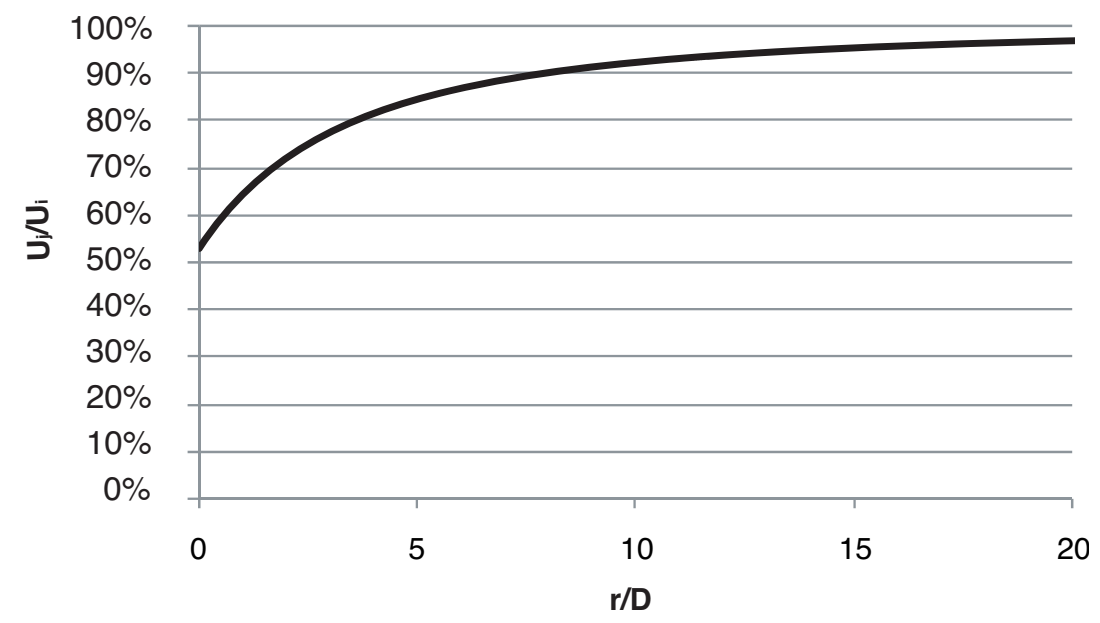

Figure 2: Illustration of wind speed deficit using modified PARK model

Magnusson and Smedman (1999) define the thrust coefficient $\left(c_{t}\right)$ shown in Equation 2 and the wake decay constant $(\tan (\mathrm{a}))$ shown in Equation 3.

$$
\begin{gathered}
c_{t}=\frac{2 T}{\rho U_{i}^{2} A} \\
\tan (\alpha)=\frac{1}{2 \ln \left(h / z_{o}\right)}
\end{gathered}
$$

As an example of the application of the model, Figure 2 illustrates the modified PARK model in terms of wind speed deficit as a function of the distance downwind (measured as the number of turbine rotor diameter lengths). Values for the constants are chosen as follows: $\tan (\alpha)=$ 0.075 (as recommended in WASP for onshore cases (WASP, 2011)) and $c_{t}=0.72$ (representative of a Vestas V80 turbine [Jensen, 2004] at typical wind speeds in our test case).

In the PARK model as $r$ tends to infinity, the wind speed returns to the original incident wind speed. This work develops an interference value $I_{i j}^{d}$ which is the loss of power caused by the interference of an upwind turbine $i$ on a downwind turbine $j$ for a given wind direction $d$. It is important to account for the velocity deficit caused by turbine interference since the power production at any location scales with the cube of the velocity at that location.

The PARK model is designed for a situation when wind flows from a single wind direction, but does not have the capability to take into account the fact that wind flow from several directions at turbine $i$ may cause interference on a turbine at location $j$. The PARK model also does not take into account the effects of wind velocity variation due to terrain. Near wake effects are not specifically addressed in the modified PARK model however this does not preclude its use for this work since the current analysis requires a model to predict velocity deficits at length scales between turbine locations as opposed to near turbines. Any other wind velocity deficit model could be used in place of the modified PARK model for interference coefficient calculation however the modified PARK model was considered to be the most relevant and suitable model currently available.

\section{WIND INTENSITY INTERFERENCE FACTOR}

This work develops a unique wind intensity interference factor which aims to better represent the interference between two turbines. This factor is dependent on the geometrical 


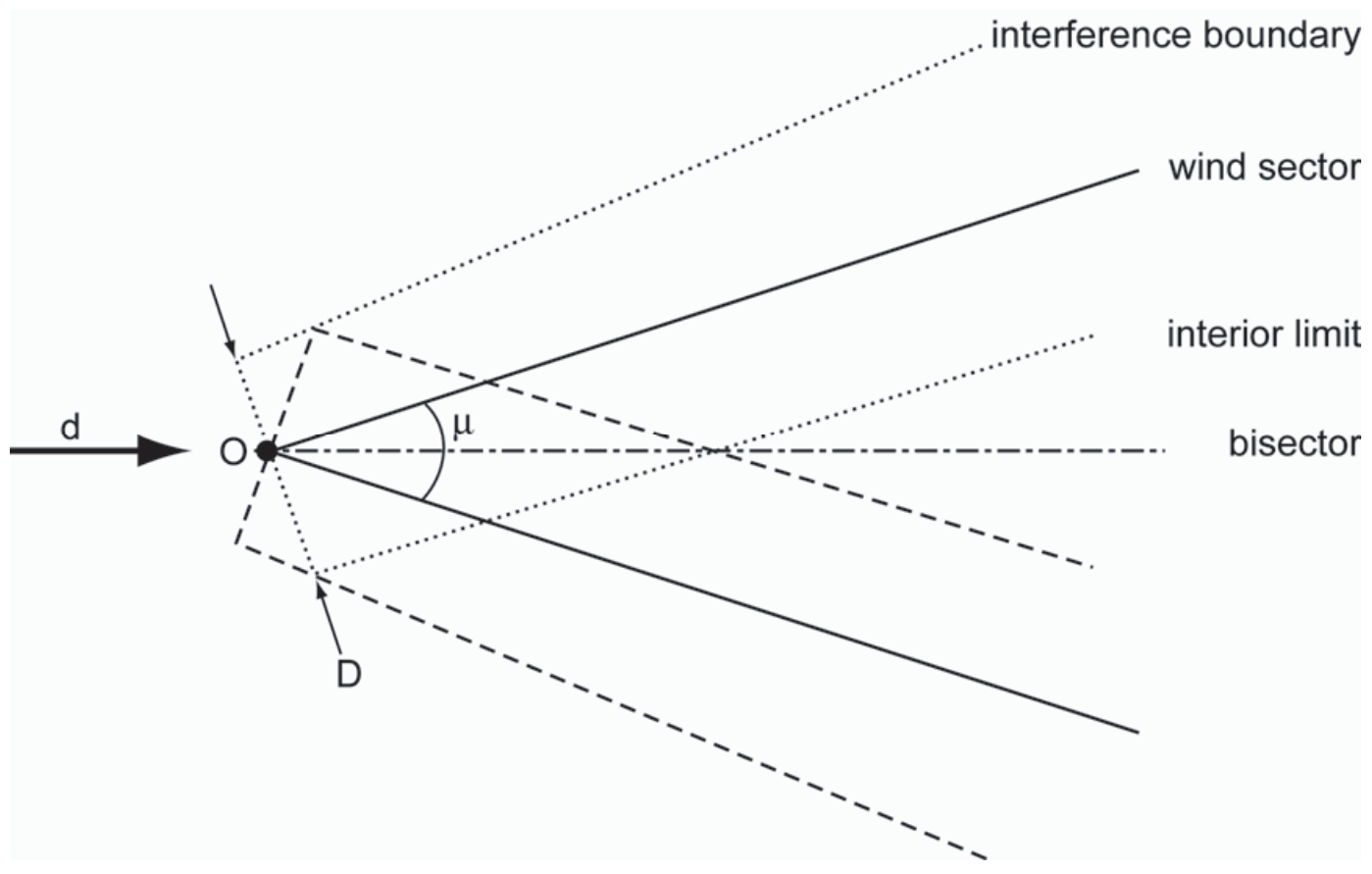

Figure 3: Schematic of turbine wake at the extremes of the wind sector

relationship between the turbines and takes into account the distribution of wind directions which occurs at each potential turbine location.

The possible wind directions are divided into $n$ sectors. Normally twelve or sixteen sectors are chosen. Each sector subtends an angle $\mu=\frac{2 \pi}{n}$ radians. We assume that within a sector any particular wind direction is equiprobable. Wind direction $d$ is defined as the statistically expected wind direction experienced within the wind sector and is the bisector of the wind sector. In Figure 3 the solid lines represent the boundaries of the wind sector. Since the wind direction can move through all angles of the wind sector, the extreme angles of the turbine wake will define the boundaries of the interference model, shown by the upper dotted and lower dashed lines. In this model there are areas outside the wind sector which are impacted by the turbine wake. Note that the boundaries of the wind sector and the interference boundary are not parallel because of the growth of the interference region defined by the $\tan \alpha$ term in Figure 1.

It should be noted that any point located within the interference boundary for a sector will be impacted by the turbine wake for a subset of wind directions within that sector. If a particular point in this domain is considered, then for any wind direction within the sector there will be a range of wind direction angles where the turbine wake includes that point. The ratio of this angle to $\mu$ is defined as the wind interference intensity factor, WIIF denoted as $\Psi(r, \theta)$. The WIIF ranges from 0 to 1 and specifies the intensity of interference on a downwind turbine for wind directions contained within a specific sector.

The radius $r$ is the distance between the upwind turbine which is located at the origin and a downwind turbine. The angle $\theta$ is the angle from the bisector of the wind sector to the downwind turbine (positive in the clockwise direction). Recall that the turbine wake angle is ! and the diameter of the turbine blades is $D$. The radial distance to a downwind turbine must be greater than a minimum separation distance between turbines as prescribed by the wind turbine manufacturer. 


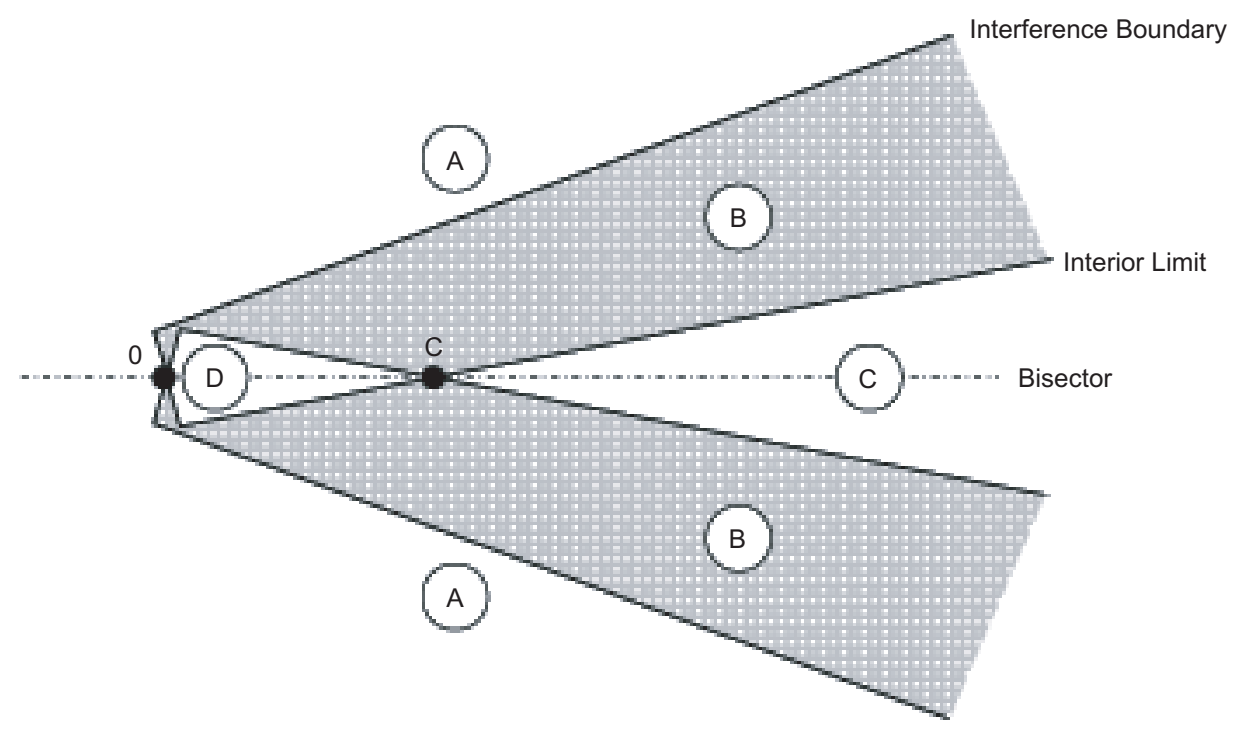

Figure 4: Illustration of the four regions with different $\Psi(r, \theta)$ calculations

There are four distinct regions of the model which require different calculations of $\Psi$. The regions are illustrated in Figure 4.

Region A is for turbines located outside the interference boundary. These turbines will have $\Psi(r, \theta)=0$.

Region B is for a downwind turbine located between the interference boundary and the interior limit. The interference boundary and interior limit are defined schematically in Figure 4 and are fully derived in the Appendix. Figure 5 illustrates a downwind turbine in region B, where a downwind turbine is located at point $Z$. The dark line between the bisector and the wind sector to the left of point $O$ is perpendicular to the turbine blades. $\Psi$ is the fraction of the

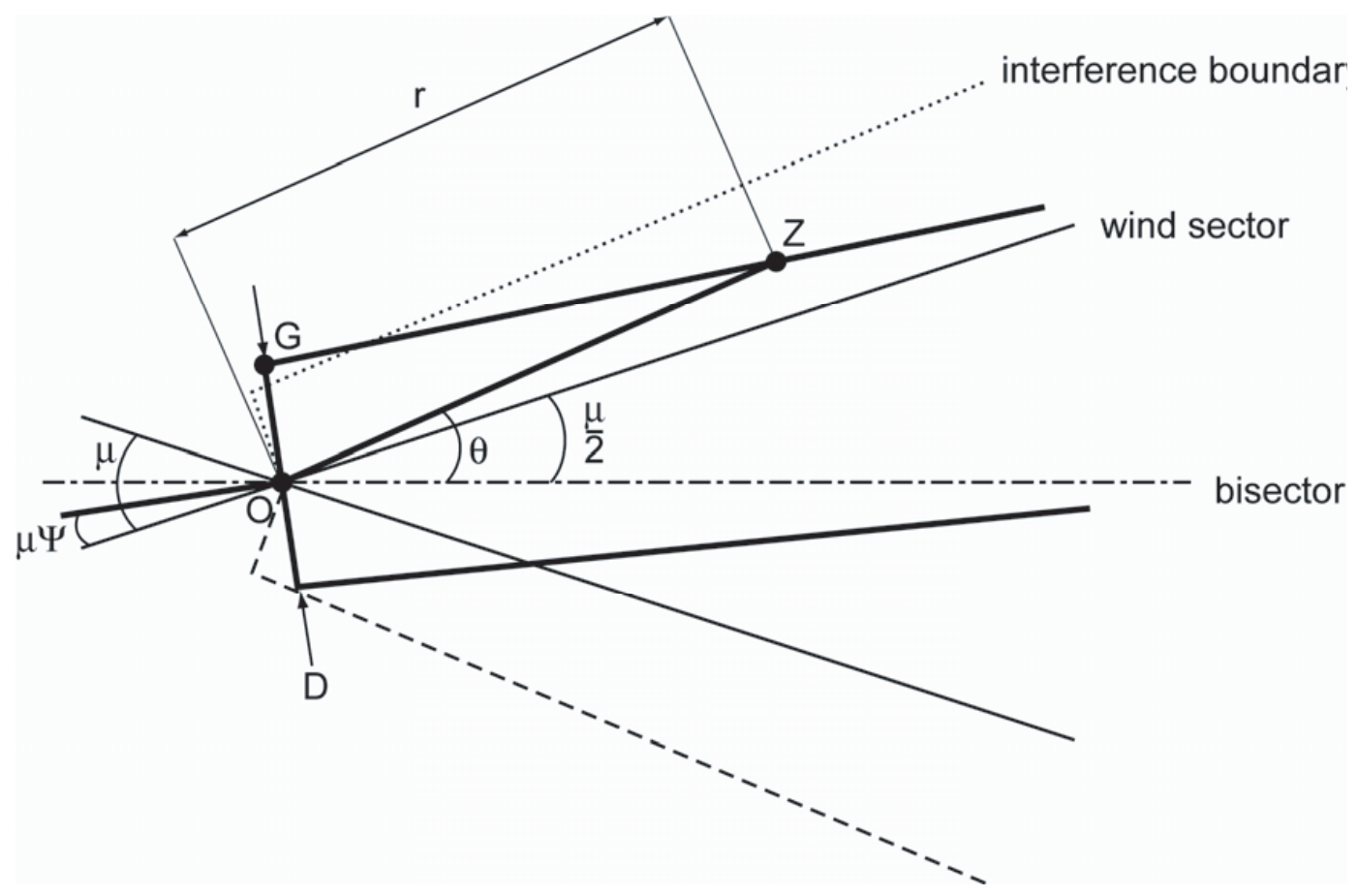

Figure 5: Schematic of the turbine wake when a downwind turbine is located at a point $\mathrm{Z}$ in region $\mathrm{B}$ 
wind sector $\mu$ in which the wind direction would cause interference on a turbine located at point $Z$ if a turbine was also located at point $O$.

The angle between the bisector and $|O G|$ is

$|\theta| \angle Z O G=|\theta|+\pi-(\angle O G Z+\angle G Z O)$

$$
=|\theta|+\pi-\left(\left(\frac{\pi}{2}+\alpha\right)+\left(\arcsin \left(\frac{D \cos (\alpha)}{2 r}\right)\right)\right)=\frac{\pi}{2}+|\theta|-\xi(r)
$$

where

$$
\xi(r)=\alpha+\arcsin \left(\frac{D \cos \alpha}{2 r}\right)
$$

Note that $\angle G Z O$ can be obtained in terms of $D, r$ and $\alpha$ by application of the sine rule to a triangle with vertices at points $\mathrm{G}, \mathrm{Z}$ and $\mathrm{O}$.

By complementary angles the angle following relationship holds:

$$
\left(\frac{\pi}{2}+|\theta|-\xi(r)\right)-\frac{\pi}{2}=\frac{\mu}{2}-\mu \Psi
$$

Therefore

$$
\Psi(r, \theta)=\frac{1}{\mu}\left(\frac{\mu}{2}+\xi(r)-|\theta|\right)
$$

Region $\mathrm{C}$ represents cases in which the downwind turbine is located within the interior boundary at a radius greater than $r_{c}=|O C|$. Figure 6 illustrates a downwind turbine in Region $\mathrm{C}$ located at point $\mathrm{Z}$. The dark lines to the right of point $O$ represent the two extreme orientations of turbine wakes which encompass point $Z$ and the dark lines to the left are perpendicular to the turbine blades at the respective orientations.

The angle between the bisector and $|\mathrm{OH}|$ is $\angle \mathrm{ZOH}=\pi-(\angle \mathrm{OHZ}+\angle \mathrm{HZO})$

$$
=\pi-\left(\left(\frac{\pi}{2}+\alpha\right)+\left(\arcsin \left(\frac{D \cos \alpha}{2 r}\right)\right)\right)=\frac{\pi}{2}-\xi(r)
$$

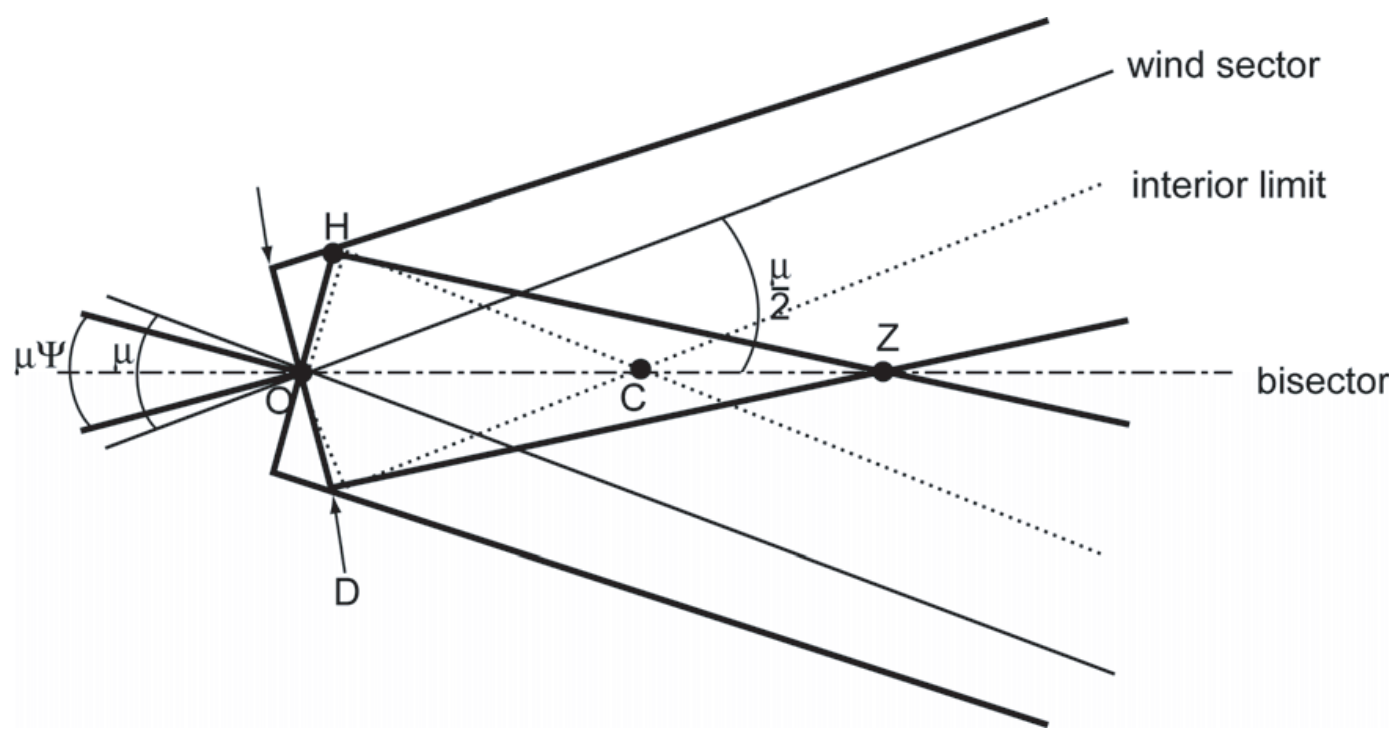

Figure 6: Schematic of turbine wake when downwind turbine is located at a point $\mathrm{Z}$ in region $\mathrm{C}$. 
$\angle H Z O$ is determined in a similar fashion to $\angle G Z O$. By complementary angles the angle between the bisector and $|O H|$ is $\frac{\pi}{2}-\left(\frac{\pi}{2}-\xi(r)\right)=\frac{\mu \Psi(r, \theta)}{2}$

therefore

$$
\Psi(r, \theta)=\frac{2 \xi(r)}{\mu} .
$$

Region D represents cases where the downwind turbine is located between the lines defining the interior boundary at a radius less than $r_{c}$. The location of point $C$ in Figure 6 is defined by $|O C|=r_{c}$. Interference will occur at any turbine located within this region for all incident wind directions within the sector. This means that $\Psi$ is always equal to 1 in this region.

A summary of the WIIF definition is shown in Equation 9.

$$
\Psi(r, \theta)=\left\{\begin{array}{lll}
\frac{1}{\mu}\left(\frac{\mu}{2}+\xi(r)-|\theta|\right), & |\varphi(r)|<|\theta| \leq|\omega(r)|, & r \geq \frac{D}{r} \\
\frac{2 \xi(r)}{\mu}, & |\theta| \leq|\varphi(r)|, & r \geq r_{c} \\
1, & |\theta| \leq|\varphi(r)|, & \frac{D}{2} \leq r<r_{c} \\
0, & \text { otherwise } &
\end{array}\right.
$$

where

$$
\begin{gathered}
\xi(r)=\alpha+\arcsin \left(\frac{D \cos \alpha}{2 r}\right) \\
\varphi(r)= \pm\left(\frac{\mu}{2}-\xi(r)\right) \\
\omega(r)= \pm\left(\frac{\mu}{2}+\xi(r)\right) \\
r_{c}=\sin (\angle C B O) \frac{|O B|}{\sin (\angle O C B)}=\frac{D \cos (\alpha)}{2 \sin \left(\frac{\mu}{2}-\alpha\right)}
\end{gathered}
$$

Figure 7 and 8 illustrate how the WIIF varies in the angular and radial directions respectively. For these figures, variables $\alpha, \mu$ and $D$ were fixed to 0.075 radians, $\pi / 6$ radians and $1 \mathrm{~m}$

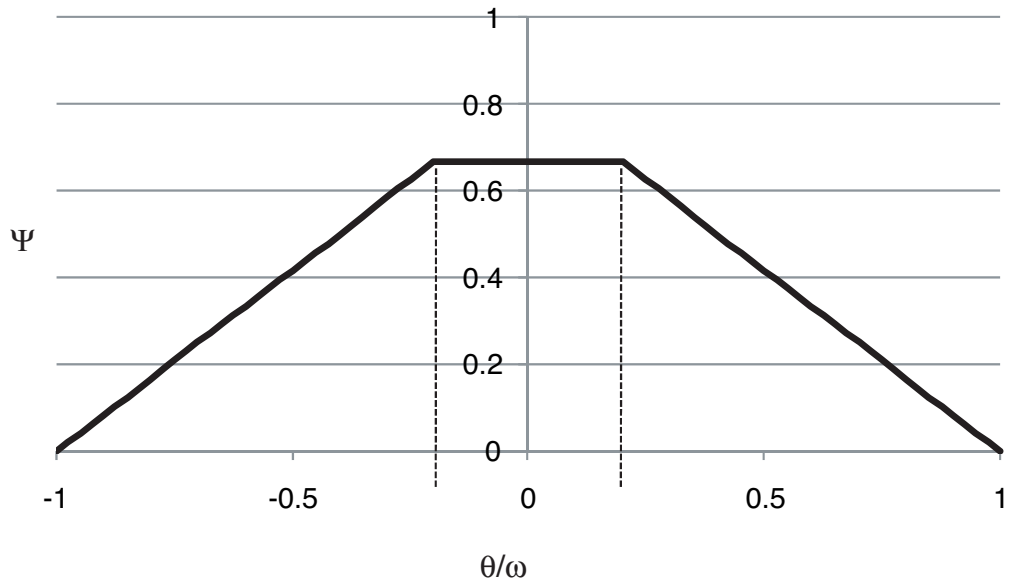

Figure 7: Angular variation of WIIF at a fixed radius $(r=5)$ 


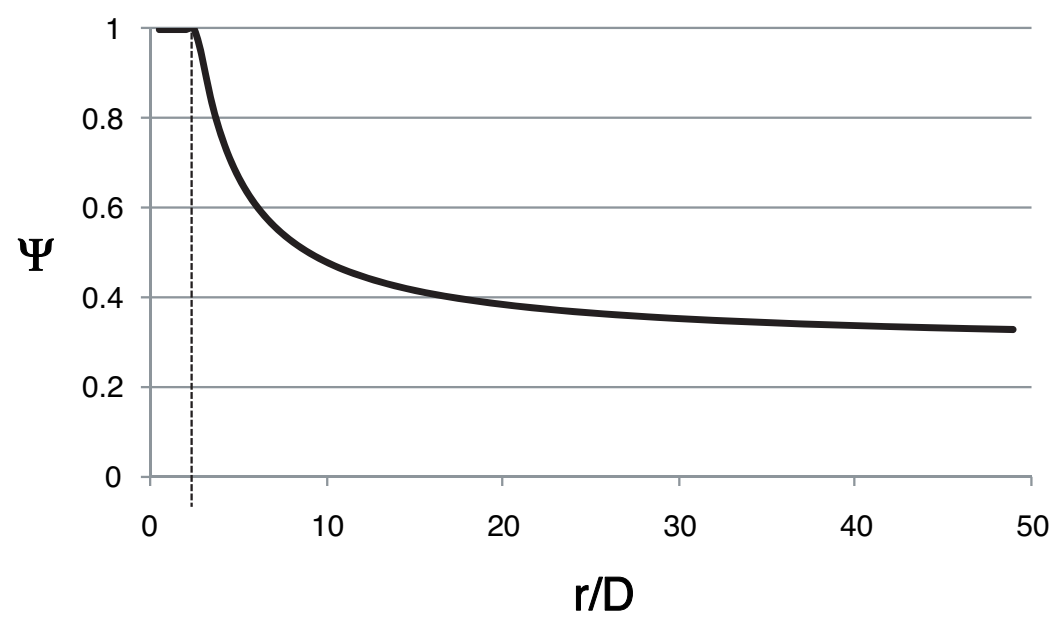

Figure 8: Radial variation of WIIF at a fixed angle $(\theta=0)$

respectively (note that $\mathrm{D}$ is chosen as $1 \mathrm{~m}$ for this illustration to normalise the values to the length of the turbine blade). In the angular direction, for a given radius $\left(r>r_{c}\right)$, the WIIF increases linearly from zero at the interference boundary through to a maximum value at the interior limit. In the radial direction, for a given angle, the WIIF has the maximum value of one for $\left(\frac{D}{2} \leq r \leq r_{c}\right)$, then decreases with distance away from the origin.

\section{INTERFERENCE COEFFICIENT}

The wind intensity interference factor developed in the previous section was developed to take account of the fact that some wind directions within a sector may cause interference on a downstream turbine (located at a position defined by $r, \theta$ ), while other wind directions do not. This factor is incorporated into an interference coefficient for use in wind farm optimization models. The interference coefficient, $I_{i j}^{d}$, is the loss of power $(P)$ caused by the interference of an upwind turbine $i$ on a downwind turbine $j$ for wind in a sector defined by direction $d$. The interference coefficient also takes account of variability in the wind speed, $U_{i}$, by assuming that the incident wind velocity follows a Weibull distribution with a probability distribution function $F_{i}^{d}$.

Other probability distributions (apart from the Weibull distribution) could be applied at this stage if desired. The interference coefficient is defined by Equation 9 .

$$
I_{i j}^{d}=\Psi(r, \theta)\left(\int_{0}^{\infty} P\left(U_{i}\right) F_{i}^{d}\left(U_{i}\right) d U_{i}-\int_{0}^{\infty} P\left(U_{j}\right) F_{i}^{d}\left(U_{j}\right) d U_{j}\right)
$$

Empirical evidence shows that the interference on a downwind turbine is overestimated when there is interference from multiple upwind turbines (Hansen et al, 2007) as shown in Figure 9. In the wind farm layout optimization model interference from multiple upwind turbines on a downwind turbine is not treated additively. Instead constraints are developed to assign the interference a downwind turbine experiences to be that from the upwind turbine which produces the greatest amount of interference at the downwind location.

\section{A MIXED INTEGER LINEAR PROGRAMMING MODEL FOR WIND FARM LAYOUT OPTIMIZATION}

The interference coefficients derived in this work are used in a mixed integer linear programming (MILP) model for wind farm layout optimization. This model determines 


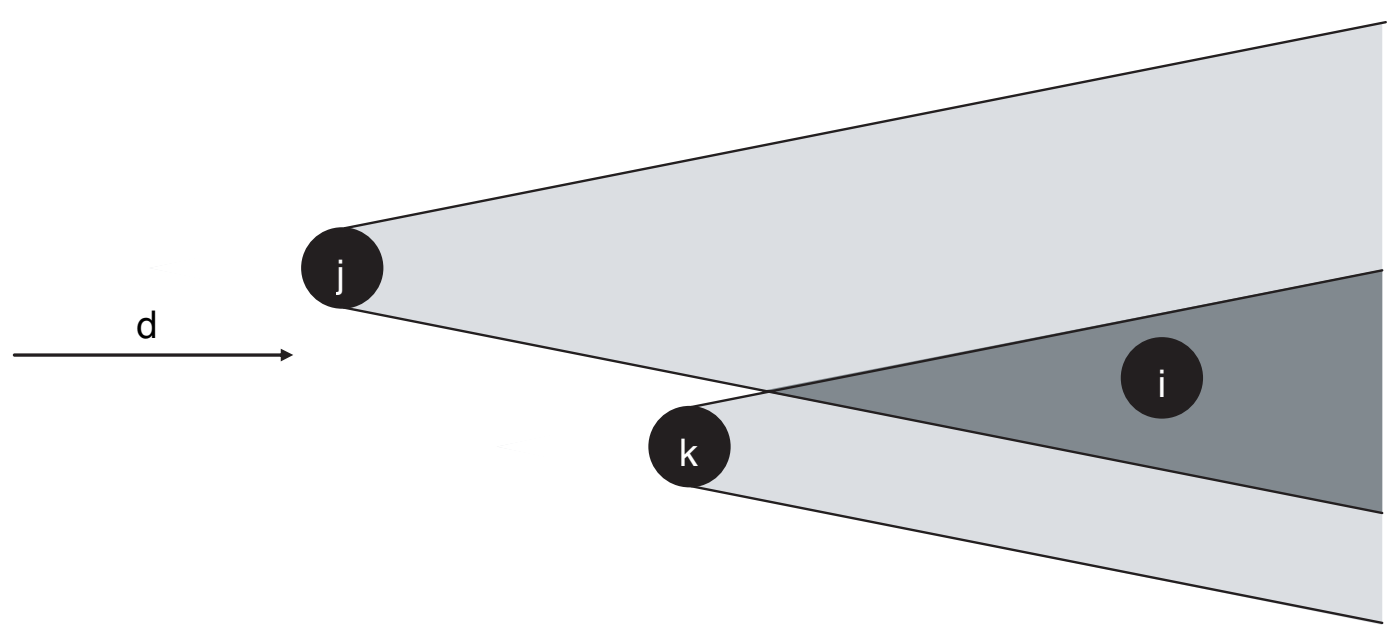

Figure 9: Schematic of the effect of multiple upwind turbines on a single downwind turbine

optimal turbine locations for a wind farm from a larger set of possible locations such that the net power output from the wind farm is maximised. Further details regarding this and other MILP models can be found in Nates (2008).

Let $V$ denote the set of possible turbine locations determined a priori. Let $S$ denote the set of wind directions. Let $Q$ denote the collection of maximal sets of turbine locations for which at most one location can be chosen due to the minimum separation distance imposed by the turbine manufacturer (see Donovan (2008) or Nates (2008) for more details). For each possible turbine location $i \in V$ let $L_{i}^{d}$ denote the set of possible turbine locations upwind from $i$ with respect to wind direction) that would cause interference at $i$ if turbines were placed in both locations. Let $w_{i}^{d}$ denote the fraction of time wind blows from direction $d \in S$. Let $P_{i}^{d}$ denote the power produced by a turbine located at $i$ in isolation. Let $I_{i j}^{d}$ denote the power loss incurred by a turbine located at $i$ due to interference by a turbine located upwind at $j \in V$ with respect to wind direction $d$. Let $b$ denote the maximum number of turbines that can be located in the wind farm.

Let the binary variable $x_{i}$ indicate that a turbine is located at $i$ when it takes value one and zero otherwise. Let the continuous variable $z_{i}^{d}$ measure the power loss at location $i$ due to interference from upwind turbines with respect to wind direction $d$.

Maximise

$$
\sum_{i \in V, d \in S} w_{i}^{d}\left(P_{i}^{d} x_{i}-z_{i}^{d}\right)
$$

Subject to

$$
\begin{gathered}
\sum_{i \in q} x_{i} \leq 1 \quad q \in Q \\
\sum_{i \in{ }_{i j}^{d}\left(x_{i}+x_{j}-1\right) \leq z_{i}^{d} \leq b} \quad i \in V, d \in S, j \in L_{i}^{d} \\
x_{i} \in\{0,1\}, z_{i}^{d} \geq 0 \quad i \in V, d \in S
\end{gathered}
$$


Equation (10) is the problem objective function which seeks to find a feasible solution to the MILP with maximum objective function value. The objective function maximises the net power output over all turbines installed at the wind farm. Equations (11-14) are the problem constraints that describe the set of feasible solutions. Constraint (11) enforces the minimum separation distance between turbines as prescribed by the turbine manufacturer. Constraint (12) determines the power loss at location $i$ due to interference from upstream turbines with respect to the wind direction $d$. Constraint (13) limits the number of turbines which can be located in the wind farm to be at most $b$. Constraint (14) ensures that the $x_{i}$ variables are binary and the $z_{i}^{d}$ variables are non-negative.

The model was applied to a hypothetical wind farm site using the demonstration data set from the WindFarmer software. That data covers an area measuring $1.7 \mathrm{~km}$ by $2.2 \mathrm{~km}$. A regularly spaced lattice of possible turbine locations at a $48 \mathrm{~m}$ spacing was superimposed on an area of $0.85 \mathrm{~km}$ by $1.1 \mathrm{~km}$ within that site. A turbine diameter of $48 \mathrm{~m}$ was assumed, with a minimum separation distance between turbines of four turbine diameters. The MILP was formulated and solved using the AMPL/CPLEX software package (version 10.0) on a $4 \mathrm{GHz}$ Pentium with 2GB of RAM. A time limit of one hour was imposed for the solution process. Imposing this limit may mean that the solution does not reach optimality, however it was observed that solutions obtained within this timeframe were generally with $2 \%$ of the optimal solution. Unlike the heuristic methods commonly used to solve these problems, MILP is able to provide a guarantee as to the quality of the solution found. If no bounds are placed on the expenditure of CPU time then the MILP will find an optimal solution.

To assess the impact of interference on the wind farm layout cases were run with and without interference. In a case which placed 25 turbines into the sample wind farm power annual production of $85,263 \mathrm{MWh}$ was achieved. The model would have predicted a further 3,641 MWh of power production if interference was not accounted for. A suitable wind farm data set is not available to us to validate this result however such comparison is envisaged in future. In a case with 26 turbines which produced an optimized turbine layout that deliver $88,280 \mathrm{MWh}$. This layout involved predicted power losses due to interference of 3,943 MWh. The model is a robust tool to predict the incremental value of adding additional turbines to the wind farm.

The MILP approach itself was compared to the heuristic methods used in WindFarmer by comparing the wind farm yields predicted from the MILP model's layout of turbines to the yield predicted by Windfarmer. Cases with 10 to 25 turbines were used in the hypothetical wind farm site (which is a demonstration data set from Windfarmer). In all cases the yield from the MILP approach was 2 to $3 \%$ greater than that predicted by Windfarmer.

\section{CONCLUSION}

This work has developed a wind intensity interference coefficient which quantifies the expected power loss due to interference on a downwind turbine caused by an upwind turbine. This factor is evaluated for every wind direction and turbine pair. The wind intensity interference coefficient is then used in a MILP model to optimize the layout of wind turbines within the domain of a wind farm. The MILP formulated in this work was an efficient and effective tool for this optimization. A test case which placed 25 wind turbines into a sample wind farm showed that ignoring wind turbine interference would over estimate the power production from this farm by 3,641 MWh. The MILP formulation can guarantee the quality of the solution which is found and outperformed predictions made by the heuristic method incorporated into the Windfarmer software. 


\section{REFERENCES}

1. Magnusson, M. and A.-S. Smedman (1999). "Air Flow Behind Wind Turbines.” Journal of Wind Engineering and Industrial Aerodynamics 80: 169-189

2. WASP software description, http://www.wasp.dk/Products/WAsP/WakeEffect Model.html, retrieved March 14 ${ }^{\text {th }}, 2011$

3. Jensen, L.E., Mørch, C., Sørensen, P.B and K.H. Svendsen, "Wake Measurements from the Horns Rev wind farm”, 2004 European Wind Energy Conference, 22-25 November, London

4. GH(2006), GH Windfarmer: The Wind Farm Design Software

5. Hansen, K., R. Barthelmie, et al. (2007). Flow and Wakes in Complex Terrain and Offshore: Model Development and Verification in UpWInd. European Wind Energy Conference \& Exhibition, Milan, Italy.

6. Jensen, N. O. (1983). A Note on Wind Generator Interaction. Roskilde, Riso National Laboratory.

7. Nates (2008) “Improved Models of Wind Farm Layout Optimisation”, Part 4 Project Report, Department of Engineering Science, University of Auckland

8. Donovan (2008) "Whither the Wind Blows: Wind Flow Modelling and Wind Farm Layout Optimisation", ME thesis, University of Auckland

\section{ACKNOWLEDGEMENT}

Jennifer De Leon is gratefully acknowledged for unwittingly helping the first author find the energy required to finalise this manuscript.

\section{APPENDIX}

Figure 10 depicts the a geometrical construction used to determine the boundary of the interference region, $\omega(r)$, In this figure point $X$ is a distance $r \geq \frac{D}{2}$ from the origin. A turbine located at a point beyond $X$ (in the angular direction, at angles greater than $\omega(r)$ ) would not receive interference from a turbine located at the origin.

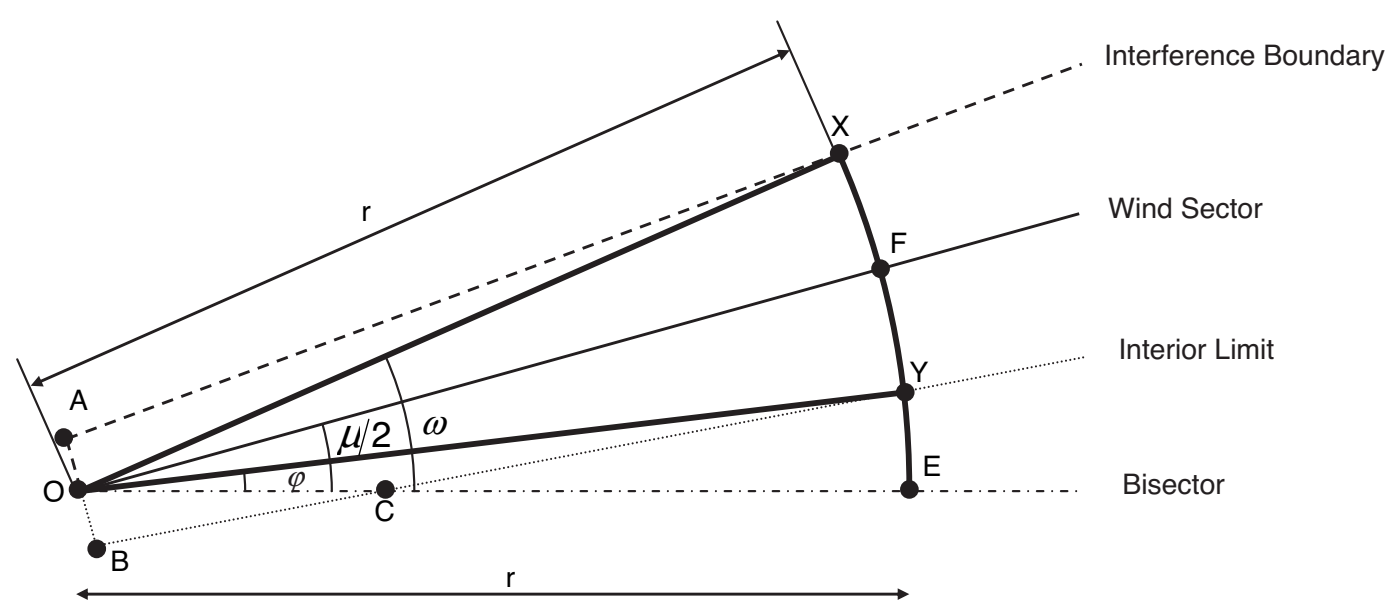

Figure 10: Determination of the interference region boundary and interior limit 